\title{
License Plate Recognition System using Back Propagation Neural Network
}

\author{
Vijay Laxmi \\ M.Tech(CSA) Scholar \\ Dept. of Computer Sc. \& Applications \\ Chaudhary Devi Lal University, Sirsa- 125055, India
}

\author{
Harish Rohil, Ph.D \\ Assistant Professor \\ Dept. of Computer Sc. \& Applications \\ Chaudhary Devi Lal University, Sirsa-125055, India
}

\begin{abstract}
Due to rapid development of technology and increasing use of vehicles, license plate recognition system has numerous applications. In present world, crimes are increasing day by day with a rapid speed and criminals use vehicles in crimes. When we go at a crowded place, we see that people does not follow traffic rules during driving due to which many road accidents occur. So we have designed a license plate recognition system to overcome these problems. Identifying the license plate of a vehicle involved in crimes can help to nab the criminals. In recent years, template matching was used for license plate recognition but it is sensitive to noise. So, neural networks are used for recognition. In this paper, Haar wavelet and back propagation neural network are used for license plate detection and feature extraction of license plate characters. The proposed system has been implemented in MATLAB.
\end{abstract}

\section{Keywords}

LPRS, Character Segmentation, Character Recognition, Back Propagation Neural Network.

\section{INTRODUCTION}

License plate recognition systems have received a lot of attention from the research community. With the rapid growth in the number of vehicles, there is a need to improve the existing systems for identification of vehicles. A fully automated system is in demand in order to reduce the dependency on labor. The Automatic Number Plate Recognition (ANPR) was invented in 1976 at the Police Scientific Development Branch in the UK. However, it gained much interest during the last decade along with the improvement of digital camera and the increase in computational capacity. It is simply the ability to automatically extract and recognition a vehicle number plate's characters from an image. In essence it consists of a camera or frame grabber that has the capability to grab an image, find the location of the number in the image and then extract the characters for character recognition tool to translate the pixels into numerically readable character.

\subsection{Phases in License Plate Recognition System}

LPR system mainly contains four phases to recognize a vehicle license plate of any country. Steps in phases are shown in Fig 1.

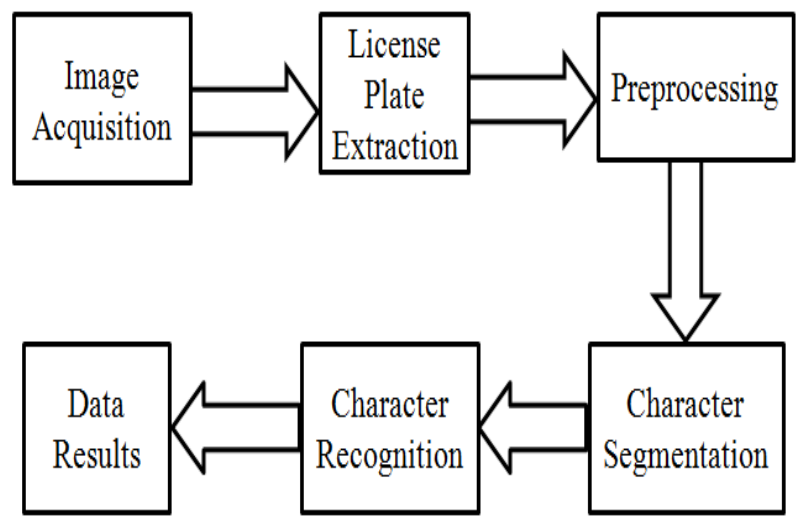

Fig 1: License Plate Recognition System Phases

These are explained as follows:

\subsubsection{Image Acquisition}

This is the first phase in an LPR system. This phase deals with acquiring an image by an acquisition method. In our proposed system, we used a high resolution digital camera to acquire the input image. The input image is $1200 \times 1600$ pixels.

\subsubsection{License Plate Extraction}

License Plate Extraction is a key step in an LPR system, which influences the accuracy of the system significantly. This phase extracts the region of interest i.e. the license plate from the acquired image. The proposed approach involves masking of a region with high probability of license plate and then scanning the whole masked region for license plate.

\subsubsection{Character Segmentation}

License Plate Segmentation, which is sometimes referred to as Character Isolation takes the region of interest and attempts to divide it into individual characters. In the proposed system segmentation is done by using bounding region method.

\subsubsection{Optical Character Recognition}

The last phase in LPR system is to recognize the isolated characters. After splitting the extracted license plate into individual character images, the character in each image can be identified. There are many methods used to recognize isolated characters. In the proposed system we are using Optical Character Recognition which is an inbuilt feature in MATLAB.

\subsection{Elements of LPR System}

LPR systems normally consist of the following units: 
Camera:-Takes image of a vehicle from either front or rear end.

Illumination:-A controlled light that can bright up the plate, and allows day and night operation. In most cases the illumination is Infra-Red (IR) which is invisible to the driver.

Frame Grabber:-An interface board between the camera and the PC that allows the software to read the image information.

Computer:-Normally a PC running Windows or Linux. It runs the LPR application that controls the system, reads the images, analyzes and identifies the plate and interfaces with other applications and systems.

Software:-The application and the recognition package.

Hardware:-Various input/output boards used to interface the external world (such as control boards and networking boards).

Database:-The events are recorded on a local database or transmitted over the network. The data includes the recognition results and (optionally) the vehicle or driver-face image file.

\section{RELATED WORK}

In license plate recognition system, a lot of work has been done by many researchers. We will explain related work in every phase of LPRS one by one. Some of which are explained as follows:

\subsection{Image Acquisition}

Image Acquisition is the first step in an LPR system and there are a number of ways to acquire images, the current literature discusses different image acquisition methods used by various authors. Comelli et. al. [1] used a CCD TV camera for image acquisition mounted on the framework of a tollgate. In such a way it is possible to frame a rear view of a passing vehicle, whose presence is reported by an ending- race sensor. Salgado et. al. [2] proposed a advanced Sensor subsystem, having a high resolution CCD camera supplemented with a number of new digital operation capabilities. Naito et. al. [3] developed a novel sensing system, which utilizes two CCDs and the prism to split an incident ray into two lights with different intensities, has been presented. One of the main features of this sensing system is that it covers wide illumination conditions from twilight to noon under sunshine. Kim et. al. [4] proposed a learning-based approach for the construction of license plate recognition system.

\subsection{License Plate Extraction}

License plate extraction is the most important phase in an LPR system. Here, we will discuss some of the previous work done during the extraction phase.

Lee et. al. [5] proposed a method to extract Korean license plate depending on the color of the plate. In this method a neural network is used for extracting color of a pixel by HLS (Hue, Lightness and Saturation) values of eight neighboring pixels and a node of maximum value is chosen as a representative color. Comelli et. al. [6] proposed a method which first applies two neural network-based filters to a color image and then it uses a post-processor to combine the two filtered images in order to locate license plates. Kim et. al. [4] presented a vehicle license plate recognition system. It consists of three modules, each of which was an integration of various vision technologies. One of them is the car extraction module which detects car in given image sequence. Tianding Chen [7] proposed a character localization method for color images. The method operates directly on the compressed image using discrete wavelet transform and neural network.

\subsection{Character Segmentation}

Many different approaches for segmentation phase have been proposed in the literature and some of them are as follows:

Seetharaman et. al. [8] used conventional technique for segmentation of characters. Once the license plate is localized and the bounding rectangle is obtained, the license plate area is hunted for a character. Salgado et. al. [2] proposed algorithms based on lateral histogram horizontal and vertical accumulations. Horizontal accumulations where used to distinguish between single and two line car-plates. Vertical accumulations where applied to separate the characters according to the so-called slopes of the vertical dynamic range. Kim et. al. [4] used neural network as filter for segmentation. To find the license plate from the candidate area, this module first applies two neural network-based filters to an input and then it uses a post-processor to combine the two filtered images in order to locate license plates. Abdullah [9] proposed a image segmentation technique using clustering approach.

\subsection{Optical Character Recognition}

This section presents the methods that were used to classify and then recognize the individual characters. The classification is based on the extracted features. These features are then classified using either the statistical, syntactic or neural approaches. Recognition module is shown in Fig 2. Some of the previous work in the classification and recognition of characters is as follows:

Khotanzand and Hong [10] used Zernike Moments by several authors for recognition of characters. Caner et. al. [11] used template matching and neural networks are the two methods frequently used in character recognition. Salgado et. al. [2] A particular character recognition strategy was developed specially suited to overcome the following problems not considered in the standard OCR approaches: small character resolution, low image contrast, motion blur, geometrical distortion and corrupted image information. Boaming Shan [12] Based on the study of plate location, the method of vertical projection information with prior knowledge is proposed to segment character and extract the statistic feature, then use the RBF neural network to recognize with feature vectors as input. 


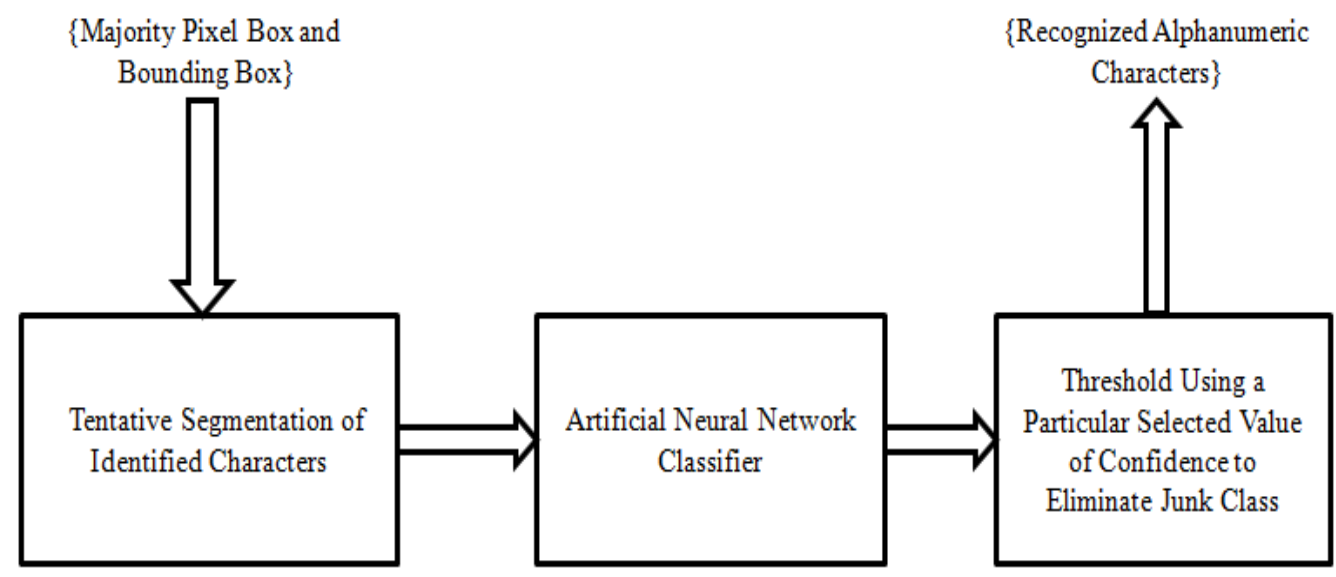

Fig 2: Recognition Module

\section{ARTIFICIAL NEURAL NETWORK}

An artificial neural network, usually called neural network is a mathematical model or computational model that is inspired by the structure and functional aspects of biological neural networks. A neural network consists of an interconnected group of artificial neurons and it processes information using a connectionist approach to computation. In most cases an artificial neural network is an adaptive system that changes its structure based on external or internal information that flows through the network during the learning phase. In an artificial neural network, simple artificial nodes, variously called neurons are connected together to form a network of nodes mimicking the biological neural network, hence the term "artificial neural network". Neural networks, with their remarkable ability to drive meaning from complicated or imprecise data, can be used to extract patterns and detect trends that are too complex to be noticed by either humans or other computer techniques. An artificial neural network is a system that receives an input, process the data and provides an output as shown in Fig 3. Input consists of data array which can be anything such as data from an image file, a wave sound or any kind of data that can be represented in an array. Once an input is presented to the neural network, and a corresponding desired or target response is set at the output, an error is composed from the difference of the desired response and the real system output. In license plate recognition system neural networks are used. In this system neural networks are used to find the accuracy. Back propagation algorithm is used to find the recognition rate.

Artificial Neural Network resembles human brain in following ways:

1) It requires knowledge through learning.

2) Artificial Neural Network knowledge is stored within interneuron connection strengths known as synaptic weights.

\subsection{The Artificial Neuron}

A single artificial neuron can be implemented in many different ways. The general mathematic definition is as showen in equation (1).

$\mathrm{y}(\mathrm{x})=\mathrm{g}\left(\sum \mathrm{w}_{\mathrm{i}} \mathrm{x}_{\mathrm{i}}\right)$

$\mathrm{x}$ is a neuron with $\mathrm{n}$ input dendrites $\mathrm{x}(0), \mathrm{x}(1)$, $\mathrm{x}(\mathrm{n})$ and one output a on $\mathrm{y}(\mathrm{x})$ and where $\mathrm{w}(0), \mathrm{w}(1)$,

..w(n) are weights determining how much the inputs should be weighted and $\mathrm{g}$ is an activation function that weights how powerful the

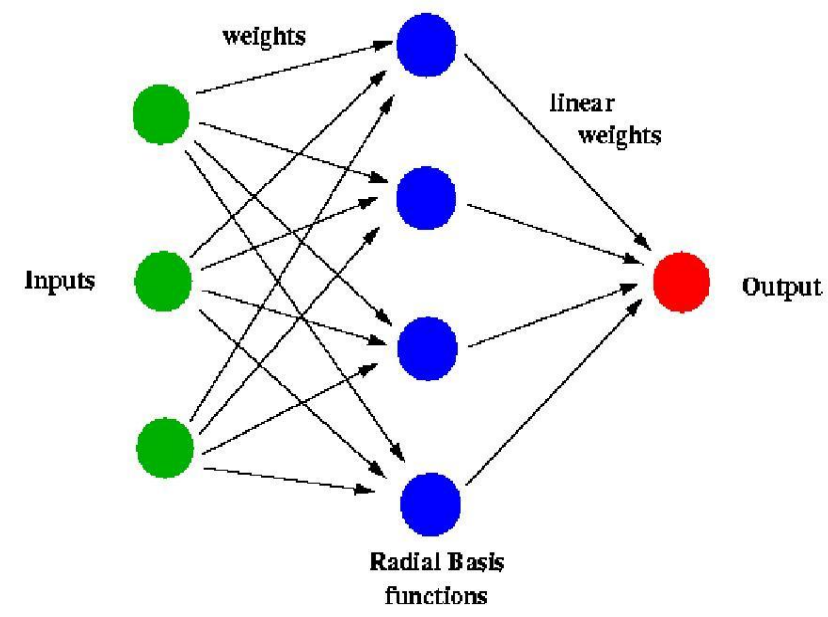

Fig 3: Block Diagram of Artificial Neural Network

output artificial neuron should mimic a real neuron; the activation function $\mathrm{g}$ should be a simple threshold function returning 0 or 1 . This is however, not the way artificial neurons are usually implemented. For many different reasons, it is preferable to have a smooth activation function. The output from the activation function is either between 0 and 1 or between -1 and 1 , depending on which activation function is used.

\subsection{Simple Neuron}

An artificial neuron is a device with many inputs and one output. The neuron has two modes of Operation: the training mode and the using mode. In the training mode, the neuron can be trained to fire (or not), for particular input patterns. In the using mode, when a taught input pattern is detected at the input, its associated output becomes the current output. If the input pattern does not belong in the taught list of input patterns, the firing rule is used to determine whether to fire or not.

\subsection{Training of Neural Network}

As neural network has to be configured such that the application of a set of inputs produces 'direct' the desired set of outputs. Various methods to set the strengths of the 
connections exist. One way is to set the weights explicitly, using a priori knowledge. Another way is to train the neural network by feeding it teaching patterns and letting it change its weights according to some learning rule. Learning of neural network is as follows:

\subsubsection{Supervised Learning}

In this type of learning network is trained by providing it with input and matching output patterns. These input-output pairs can be provided by an external teacher or by the system which contains the neural network.

\subsubsection{Unsupervised learning}

In this type of learning output unit is trained to respond to clusters of pattern within the input. In this paradigm the system is supposed to discover statistically salient features of the input population. Unlike the supervised learning paradigm, there is no a priori set of categories into which the patterns are to be classified; rather the system must develop its own representation of the input stimuli.

\subsubsection{Reinforcement Learning}

This type of learning may be considered as an intermediate form of the above two types of learning. Here the learning mach in its parameters. The self organizing neural learning may be categorized under this type of learning.

\section{PROPOSED APPROACH}

The proposed approach of license plate recognition system uses neural network for calculation of recognition time and training time.

\subsection{Neural Networks in License Plate Recognition}

In early days, template matching techniques were used for license plate recognition but these were sensitive to noise, so neural networks are used for recognition. Neural network has capability of learning, i.e. how to do tasks based on data given for training. Back propagation is supervised form of learning. In License plate recognition system, features extracted from license plate are used as input to neural networks and these are allowed to propagate forward to generate output. Learning of neural networks is done by loading targets and features extracted from license plate characters. Testing of neural network is done and parameters used for performance evaluation i.e. recognition rate and training time is computed for Haar wavelet.

\subsection{Back Propagation Neural Network}

Neural network is a mathematical model that is inspired by the structure and functional aspects of biological neural networks. First of all we have to initialize the neural network. In this approach we are using back propagation neural network. A general block diagram of back propagation neural network is shown in Fig 4.

Firstly, we have to define the training parameters:

- Number of iterations

- Number of hidden layer neurons

- Initial conditions

Back Propagation Neural Network (BPNN), is a Multilayer Neural Network which is based upon back propagation algorithm for training. This neural network is based upon

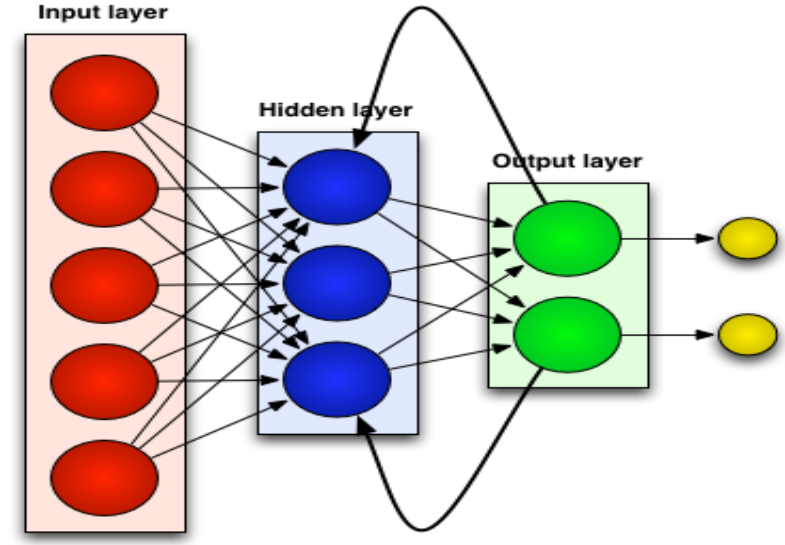

Fig 4: Back Propagation Neural Network

extended gradient descent based Delta learning rule, commonly known as Back Propagation rule. In order to train a neural network to perform some task, we must adjust the weights of each unit in such a way that the error between the desired output and the actual output is reduced. This process

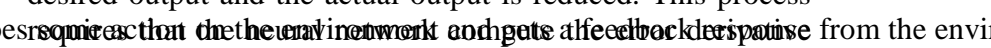
of the weights. In other words, it must calculate how the error changes as each weight is increased or decreased slightly. The back propagation algorithm is the most widely used method for determining the derivative of weights. The back propagation algorithm is easiest to understand if all the units in the network are linear. In this network, error signal between desired output and actual output is being propagated in backward direction from output to hidden layer and then to input layer in order to train the network.

Artificial neural network consists of input layer, hidden layer and output layer. Firstly, training data is fed into the input layer. It is propagated to both the hidden layer and the output layer. This process is called the forward pass. Each node in the input layer, hidden layer and output layer calculates and adjusts the appropriate weight between nodes and generate output value of the resulting sum. After that the actual output values will be compared with the target output values. The error between these outputs will be calculated and propagated back to hidden layer in order to update the weight of each node again. This process is called learning. The error calculated is shown as in equation (2).

$\mathrm{E}=1 / 2\left[\mathrm{~d}_{\mathrm{k}}-\mathrm{y}_{\mathrm{k}}\right]^{2}$

Where $d_{k}$ is desired output of neural network.

The network will iterate over many cycles until the error is acceptable. After the training phase is done, the trained network is ready to use for any new input data. During the testing phase, learning does not occur. The testing input is fed into the input layer and the feed forward network will generate results based on its knowledge from trained network. The algorithm computes each derivative of error by first computing the rate at which the error changes as the activity level of a unit is changed. For output units, the error is simply the difference between the actual and the desired output. To compute the error for a hidden unit in the layer just before the output layer, we first identify all the weights between that hidden unit and the output units to which it is connected. We then multiply those weights by the error of those output units and add the products. This sum equals the error for the chosen hidden unit. After calculating all the errors in the hidden layer just before the output layer, we can compute in like fashion 
the EAs for other layers, moving from layer to layer in a direction opposite to the way activities propagate through the network. Once the error has been computed for a unit, it is straight forward to compute the derivative of error for each incoming connection of the unit. Back propagation is most generally used algorithm in neural network. The testing input is given to input layer and it will provide results according to knowledge it get from training.

License plate recognition algorithms were implemented using wavelet and neural network in MATLAB and results were obtained using recognition rate and time required. Flow chart of recognition system is show in Fig 5.

License plate detection is the first step of License plate recognition system and directly related to the speed and accuracy of license plate recognition. In this approach, extraction of edge of gray scale image is used to locate the license plate accurately. Normalization of gray scale image is done. Sharpening filter is used for masking. Wavelet transform is done using different wavelet filters. Edge detection is done using sobel operator to extract the edge image of the original gray image. Vertical edge image is achieved by using sobel operator.

Wavelet Transform is done using Wavelet Filter. In this process, image is firstly decomposed using Wavelet toolbox's function wavedec 2 and four details of an image are formed. These four details are approximation, vertical, horizontal and diagonal details. These four details give complete information of image because image is decomposed from all sides i.e. horizontally, vertically and diagonally. In this paper, Haar wavelet filter is used for this work. Based on these four information, back propagation neural network is trained and performance parameters are calculated.

\subsection{Experimental Evaluation}

We have conducted a number of experiments for evaluation of our proposed system. One of them is described with details below:

Image shown in Fig $\mathbf{6}$ is captured by mobile camera with 3.5 megapixel and given as first input in MATLAB. This is the image for which we have done recognition of character. Image shown in Fig 7 gives four details of input image using Haar wavelet i.e. approximation, vertical, horizontal and diagonal details. With the help of these details we detect license plate as shown in in Fig 8.

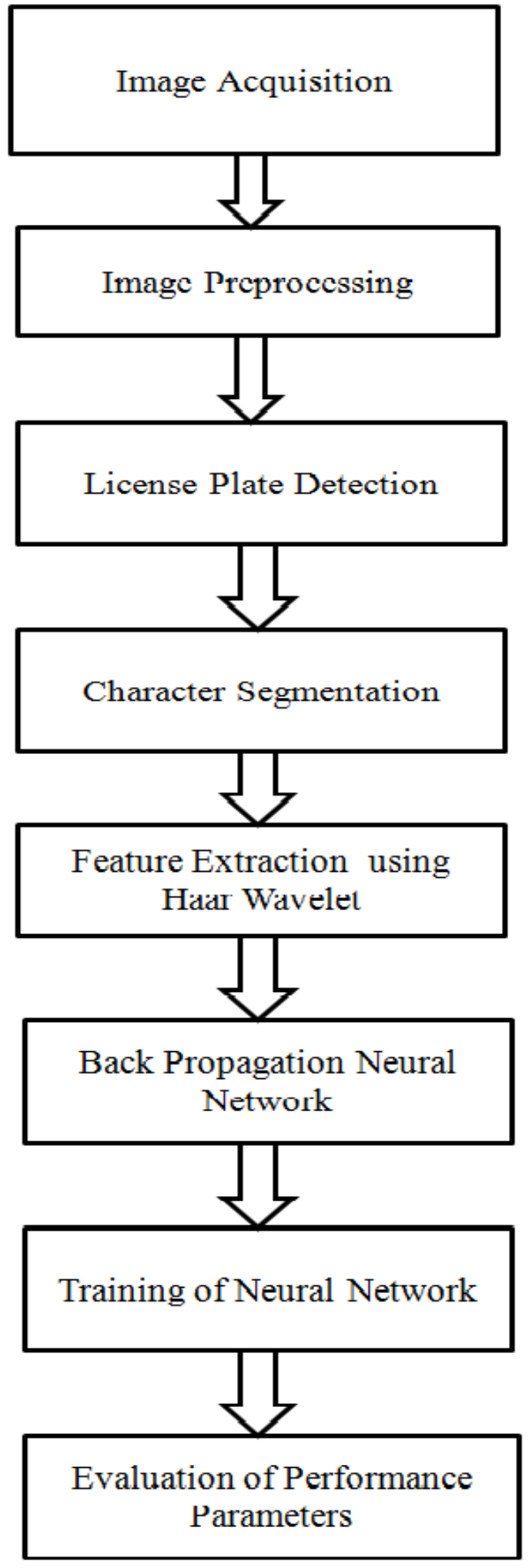

Fig 5: Flow Chart of License Plate Recognition System 


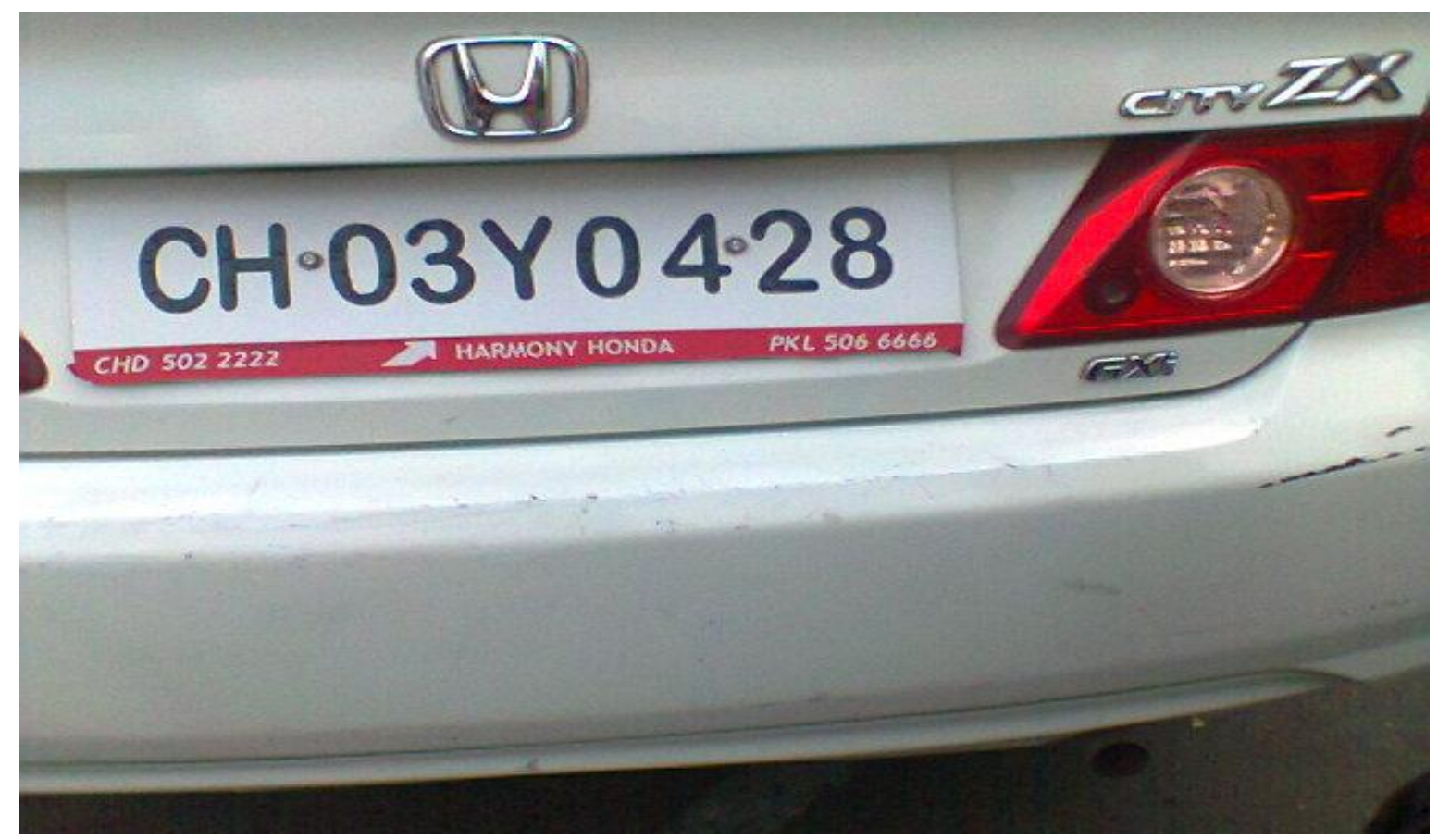

Fig 6: Input Image

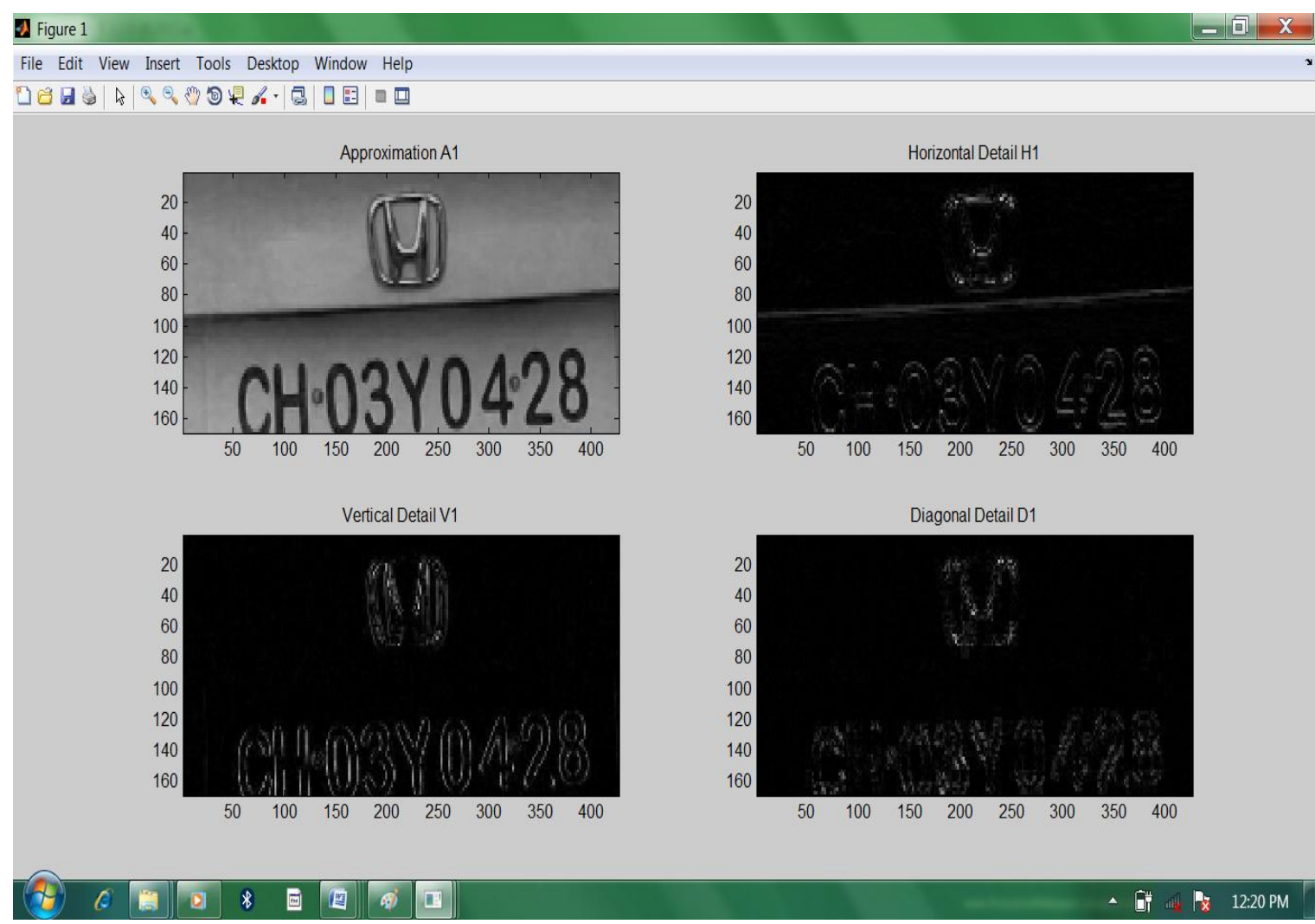

Fig 7: Details Using Haar Wavelet 


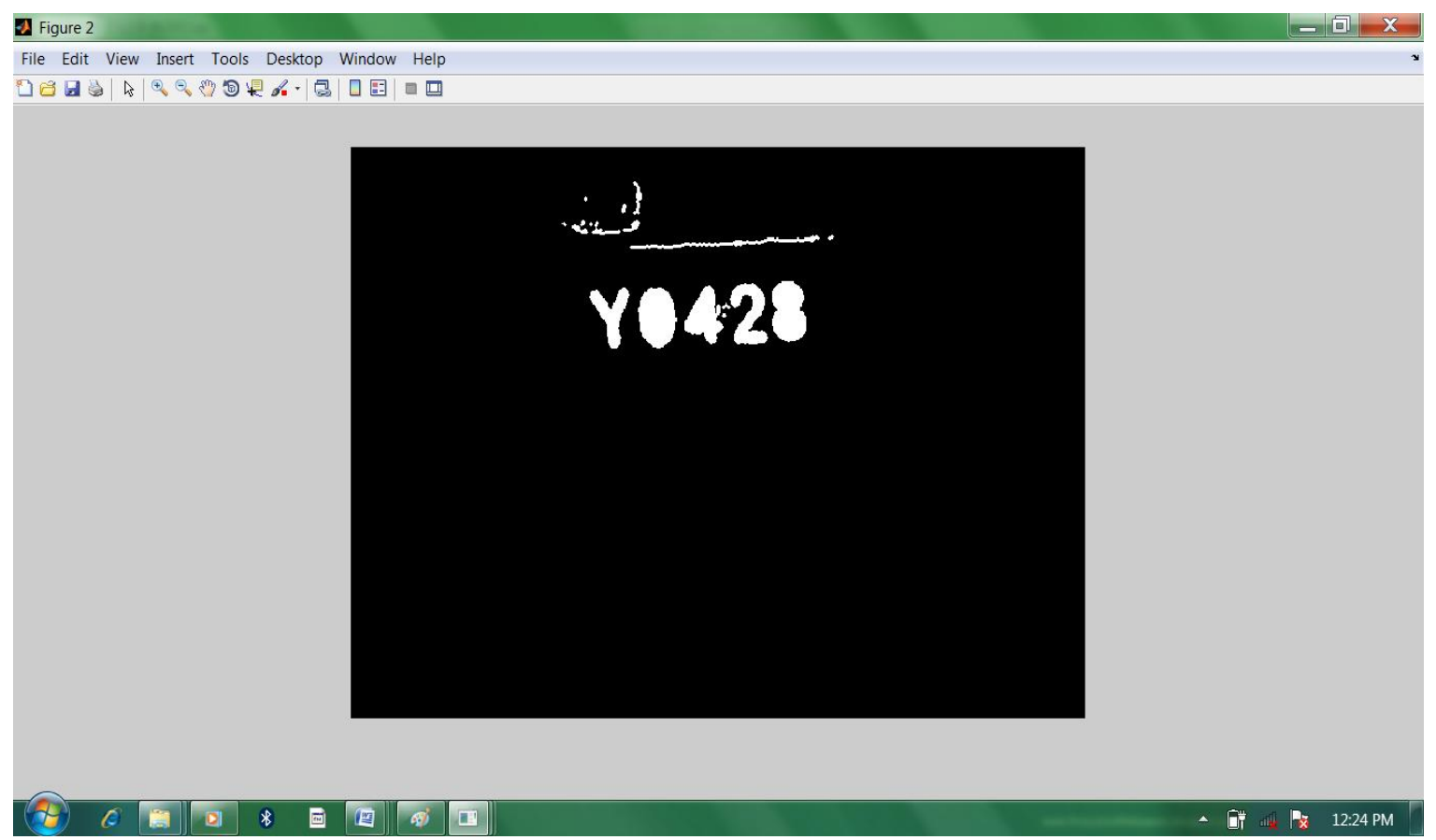

Fig 8: License Plate Detection

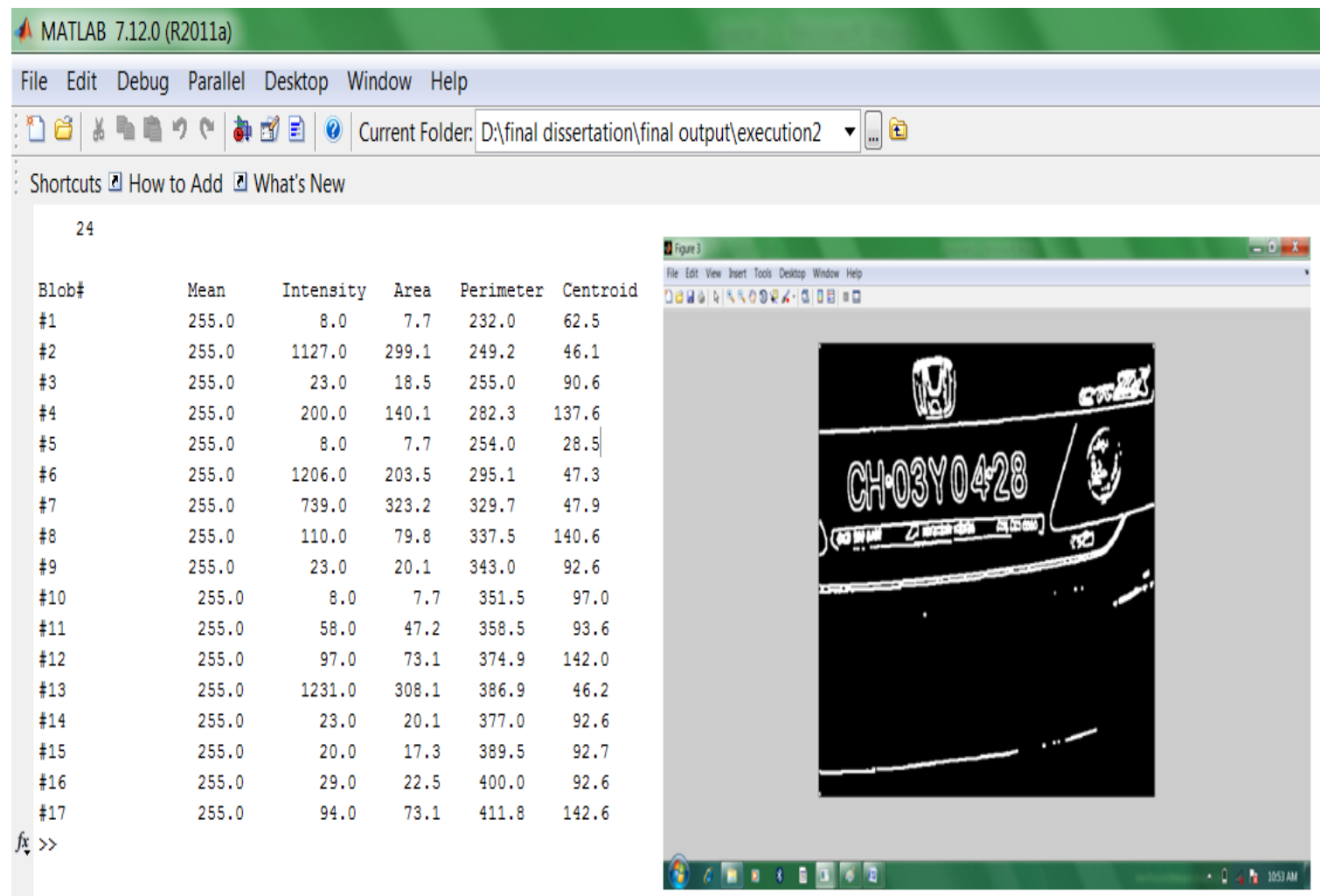

Fig 9: Character Segmentation with Feature Extraction

We extract statistical features e.g. blobs, mean, intensity, area, perimeter, centroid etc. by using Haar wavelet as shown in Fig 9. Our final result i.e. character segmentation of license plate with workspace area is shown in Fig 10. After that features of license plates are extracted and these features are used as input to neural networks for calculating recognition rate and training time. 


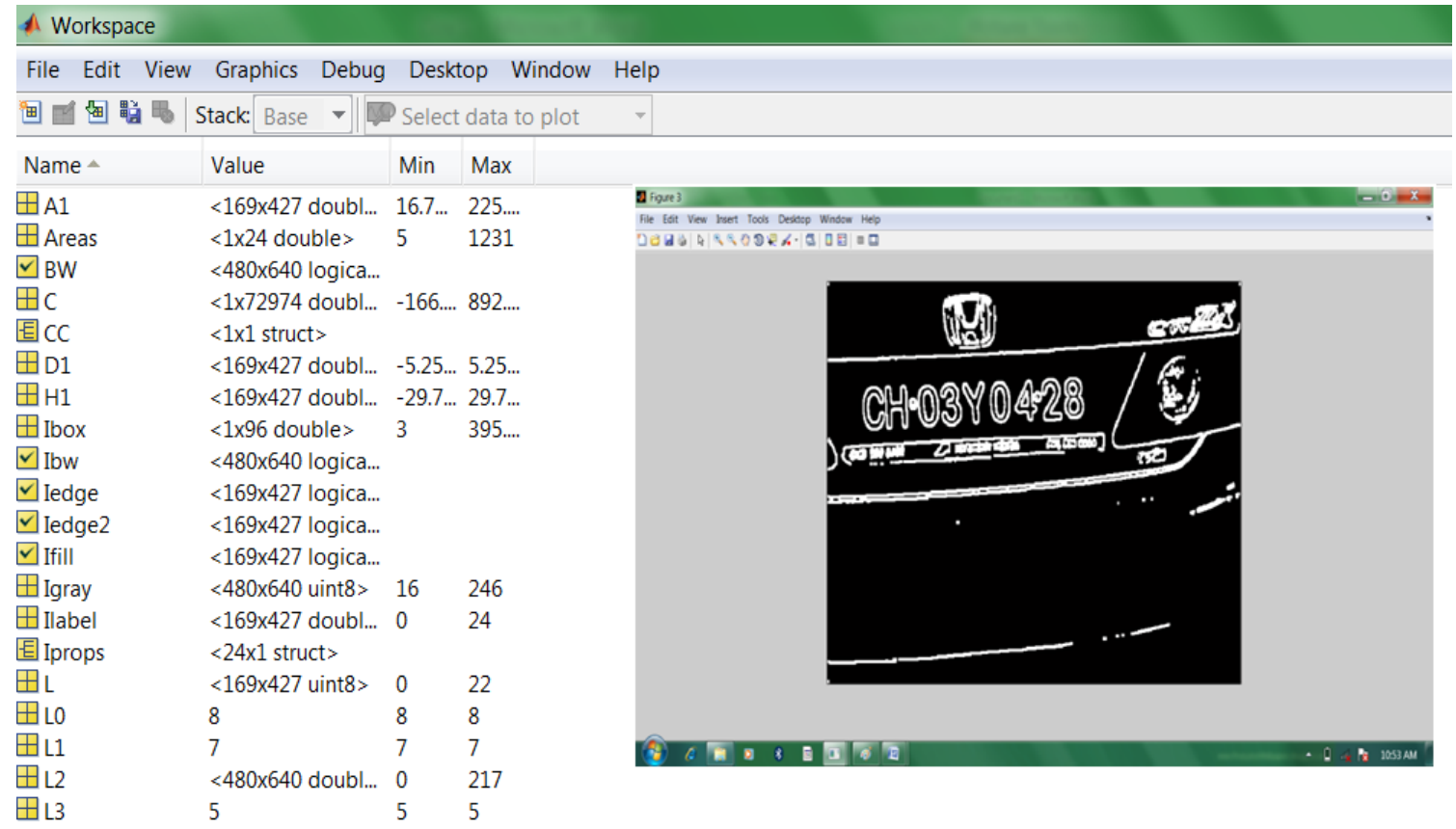

Fig 10: Character Segmentation of License Plate with Workspace Area

\subsubsection{Recognition Rate}

Recognition rate can be defined as percentage of correctly classified samples over all samples. Features extracted from license plate are taken as input to neural networks. Training of neural network is done by making database of these extracted features.

Recognition rate is computed by neural networks giving these features as input and targets are also loaded. Recognition rate is calculated from the confusion matrix and it is given by equation (3).

$$
\text { Recognition rate }=(\mathrm{TP}+\mathrm{TN}) /(\mathrm{TP}+\mathrm{TN}+\mathrm{FP}+\mathrm{FN})
$$

Where TP (True Positive) is percentage of samples correctly classified, TN(True Negative) is percentage of samples correctly classified from any other class, FP(False Positive) is percentage of members of other classes which are incorrectly classified, False Negatives (FN) is percentage of members of the class which are incorrectly classified as not belongs to same class.

Table 1. Recognition Rate for different number of Images

\begin{tabular}{|c|c|}
\hline Number of Images & Recognition Rate(\%) \\
\cline { 2 - 2 } & Haar \\
\hline 10 & 85 \\
\hline 15 & 85.5 \\
\hline 20 & 86 \\
\hline 25 & 87.5 \\
\hline 30 & 88 \\
\hline
\end{tabular}

Recognition rate is computed using different number of images in training dataset. Recognition rate for wavelet Haar is computed. From Table 1, it is concluded that recognition rate is different when different number of images are taken for training. As number of images is increased for training, recognition rate is also increased.

\subsubsection{Training Time}

The time required for whole program is computed in three parts. Firstly, time is computed for detection, character segmentation and feature extraction of license plate. After feature extraction, training of neural network is done and training time is computed. Training time is the time required for training of neural networks. It is computed for Haar wavelet by giving features extracted using as inputs to neural networks. At the end, total time is computed by taking summation of time taken for detection, character segmentation, feature extraction of license plate and training time required for training of neural networks.

Training time is computed using different number of images in training dataset. Training time for wavelet Haar is computed with the help of back propagation neural network as shown in Table 2. Training time increases when no of images in database increases. E.g. for 30 images, training time measured is 42 seconds.

Table 2. Training Time for Different Number of Images

\begin{tabular}{|c|c|}
\hline Number of Images & Training Time(in sec.) \\
\cline { 2 - 2 } & Haar \\
\hline 10 & 31 \\
\hline 15 & 35 \\
\hline 20 & 38 \\
\hline 25 & 40 \\
\hline 30 & 42 \\
\hline
\end{tabular}




\section{CONCLUSION}

The purpose of this paper is to provide an approach which can recognize license plate number of a vehicle with optimum accuracy. In the proposed license plate recognition system, back propagation neural network and Haar wavelet are used for license plate detection and character segmentation. Different Statistical measures are computed for detecting license plate using Haar wavelet. These measures are used as input to neural network. Recognition rate and training time are computed by taking different number of images in training database. As number of license plate images is increased in database, time taken for training of neural networks also increases. According to the experimental results, the proposed method can successfully locate text region from complex environment. It demonstrates the feasibility of this system.

\section{ACKNOWLEDGEMENTS}

I sincerely thanks to Dr. Harish Rohil, assistant professor, Chaudhary Devi Lal University, sirsa, Haryana(INDIA) for his guiadance and support.

\section{REFERENCES}

[1] Paolo Comelli, Paolo Ferragina, Mario Notturno Granieri, and Flavio Stabile, "Optical Recognition of Motor Vehicle License Plates", IEEE Transactions on Vehicular Technology, Vol.44, No.4, November 1995.

[2] Luis Salgado, Jose M. Menendex, Enrique Renddn, and Narciso Garcia, "Automatic Car Plate Detection and Recognition through Intelligint Vision Engineering”, 07803-5247-5/99 IEEE 1999

[3] Takashi Naito, Toshihiko Tsukada, Keiichi Yamada, Kazuhiro Kozuka, and Shin Yamamoto, "Robust License Plate Recognition Method for Passing Vehicle Under Outside Environment", Transactions on Vehicular Technology", Vol.49, No.6, November 2000.
[4] K.K.Kim, K.I.Kim, J.B.Kim, H.J.Kim, "Learning Based Approach for License Plate Recognition", 0-78036278-0/00 IEEE 2000.

[5] Eun Ryung Lee, Pyeoung Kee kim, and Hang Joon Kim, "Automatic Recognition of a Car License Plate Using Color Image Processing”, 0-8186-6950-0/9 IEEE 1994.

[6] Paolo Comelli, Paolo Ferragina, Mario Notturno Granieri, and Flavio Stabile, "Optical Recognition of Motor Vehicle License Plates", IEEE Transactions on Vehicular Technology, Vol.44, No.4, November 1995.

[7] Tianding Chen, "License Plate Text Localization Using DWT and Neural Network", Zhejiang Province Nature Science Foundation under Grant Y107411.

[8] Vivek Seetharaman, Vidhya Sudhan L Nathan, “ License Plate Recognition System Using Hybrid Neural Networks", 0-7803-8376-1/04 IEEE 2004.

[9] Siti Norul Huda Sheikh Abdullah, "License Plate Recognition Using Multilayer Neural Networks", ICOCI 2006.

[10] Alireza Khotanzad and Yaw Hua Hong, “ Invariant Image Recognition by Zernike Moments", IEEE Transaction on Pattern Analysis and Machine Intelligence, Vol.12, No.5, May 1990.

[11] Hakan Caner, H. Selcuk Gercim, and Ali Ziya Alkar, “ Efficient Embedded Neural - Network - Based License Plate Recognition System", IEEE Transactions on Vehicular Technology”, Vol.57, No.5, September 2008.

[12] Baoming Shan, "License Plate Character Segmentation and Recognition Based on RBF Neural Network", Second International Workshop on Education Technology and Computer Science 2010. 